\title{
New Candidate Planetary Nebulae in the IPHAS Survey: the Case of Planetary Nebulae with ISM interaction
}

\author{
Laurence Sabin $^{\mathrm{A}, \mathrm{F}}$, Albert A. Zijlstra ${ }^{\mathrm{A}}$, Christopher Wareing ${ }^{\mathrm{B}}$, Romano L. M. Corradi ${ }^{\mathrm{C}}$, \\ Antonio Mampaso $^{\mathrm{C}}$, Kerttu Viironen ${ }^{\mathrm{C}}$, Nicholas J. Wright ${ }^{\mathrm{D}}$, and Quentin A. Parker ${ }^{\mathrm{E}}$ \\ A Jodrell Bank Center for Astrophysics, School of Physics and Astronomy, University of Manchester, \\ Manchester, M13 9PL, UK \\ B Department of Applied Mathematics, University of Leeds, Leeds, LS2 9JT, UK \\ ${ }^{\mathrm{C}}$ Instituto de Astrofisica de Canarias, Tenerife, Spain \\ D Harvard-Smithsonian Center for Astrophysics, 60 Garden Street, Cambridge, MA, 02138, USA \\ E Macquarie University/Anglo-Australian Observatory, Department of Physics, North Ryde, \\ Sydney, NSW 2190 \\ F Corresponding author. Email: laurence.sabin@manchester.ac.uk
}

Received 2009 July 20, accepted 2009 December 8

\begin{abstract}
We present the results of the search for candidate Planetary Nebulae interacting with the interstellar medium (PN-ISM) in the framework of the INT Photometric $\mathrm{H} \alpha$ Survey (IPHAS) and located in the right ascension range $18-20 \mathrm{~h}$. The detection capability of this new Northern survey, in terms of depth and imaging resolution, has allowed us to overcome the detection problem generally associated to the low surface brightness inherent to PNe-ISM. We discuss the detection of 21 IPHAS PN-ISM candidates. Thus, different stages of interaction were observed, implying various morphologies i.e. from the unaffected to totally disrupted shapes. The majority of the sources belong to the so-called WZO2 stage which main characteristic is a brightening of the nebula's shell in the direction of motion. The new findings are encouraging as they would be a first step into the reduction of the scarcity of observational data and they would provide new insights into the physical processes occurring in the rather evolved PNe.
\end{abstract}

Keywords: ISM — planetary nebulae: general — surveys

\section{Introduction}

Large $\mathrm{H} \alpha$ surveys have so far allowed the detection of $\sim 3000$ planetary nebulae (PNe) in the Galaxy. The data can be principally found in the Strasbourg-ESO Catalogue (Acker et al. 1992) and the recent Macquarie-AAOStrasbourg $\mathrm{H} \alpha$ Planetary Nebula Catalogues: MASH I and II (Parker et al. 2006; Miszalski et al. 2008). Unfortunately a limitation in our understanding of this short and rather complex phase of stellar evolution lies either in the deepness of the detections realised or the type of PNe investigated. Indeed, although enormous progress has been made over the years in terms of observations, the well-studied PNe are generally bright and often young. This hampers the study of:

- PNe hidden by the interstellar medium, particularly those located at low galactic height.

- PNe with (very) low surface brightness where we find the group of old PNe.

- Very distant PNe which appear as unresolved and not recognisable as nebulae.

- PNe located in crowded areas such as the galactic plane.

Moreover, excluding these objects from global studies (morphology, abundances, luminosity function, etc.) may bias our understanding of planetary nebulae. As an illustration, few $\mathrm{PNe}$ are described in the literature as 'PNe with ISM interaction', which is the step before the complete dilution of the nebulae in the interstellar medium (Borkowski, Sarazin \& Soker 1990; Ali, El-Nawawy \& Pfeiderer 2000; Xilouris et al. 1996; Tweedy et al. 1996). The study of the interaction process would give new insights into several aspects of the PN evolution. Indeed, the density difference between ISM and PNe will affect their shape. This is expected to be observable in old objects where the nebular density declines sufficiently to be overcome by the ISM density. Other phenomena like the flux and brightness enhancement following the compression of the external shell, the increase of the recombination rate in the PN (Rauch et al. 2000), the occurrence of turbulent Rayleigh-Taylor instabilities and the implication of magnetic fields (Dgani et al. 1998) are among the physical processes which need to be addressed not only from a theoretical but also observational point of view.

The low surface brightness generally associated to PNe-ISM has for a long time prevented any deeper observation and good statistical study of these interactions, where only the interacting rim is well seen. New generations of $\mathrm{H} \alpha$ surveys have overcome this problem. 

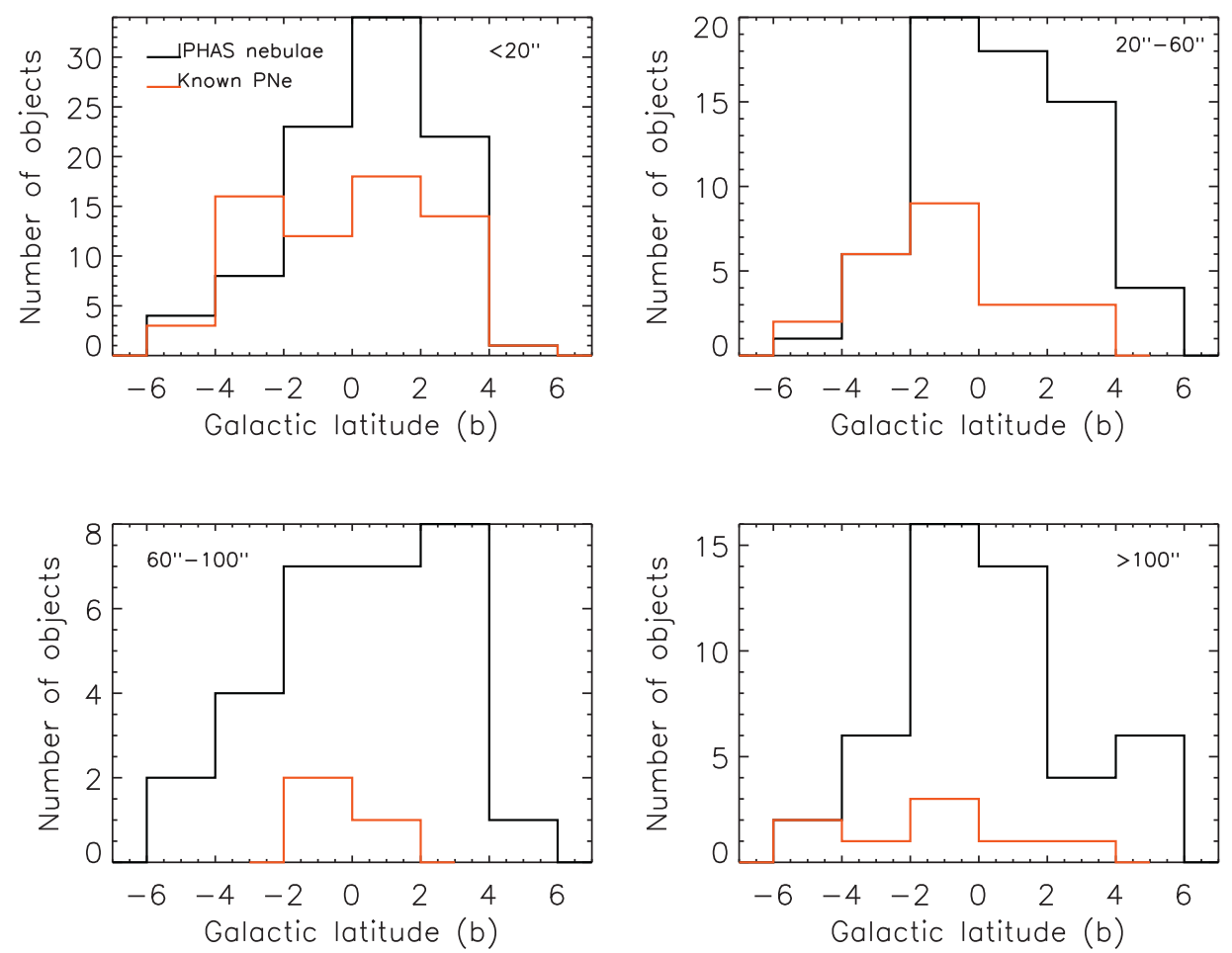

Figure 1 Galactic distribution of the IPHAS nebulae according to their size.

A perfect example is the discovery of PFP 1 by Pierce et al. (2004) in the framework of the AAO/UKST SuperCOSMOS H $\alpha$ survey (SHS) (Parker et al. 2005). This PN, starting to interact with the ISM at the rim, is very large (radius $=1.5 \pm 0.6 \mathrm{pc}$ ) and very faint (logarithm of the $\mathrm{H} \alpha$ surface brightness equal to $\left.-6.05 \mathrm{erg} \mathrm{cm}^{-2} \mathrm{~s}^{-1} \mathrm{sr}^{-1}\right)$. In order to unveil and study this 'missing PN population' in the Northern hemisphere we need surveys providing the necessary observing depth: the Isaac Newton Telescope (INT) Photometric H $\alpha$ Survey (IPHAS) is one of them and will complete the work done in the South by the SHS.

\section{IPHAS Contribution}

IPHAS is a new fully photometric CCD survey of the Northern Galactic Plane, started in 2003 (Drew et al. 2005; Gonzalez-Solares et al. 2008) and which has now been completed. ${ }^{1}$ Using the $2.5-\mathrm{m}$ Isaac Newton Telescope (INT) in La Palma (Canary Islands, SPAIN) and the Wide Field Camera (WFC) offering a field of view of $34.2 \times 34.2 \mathrm{arcmin}^{2}$, IPHAS targets the Galactic plane in the Northern hemisphere, at a latitude range of $-5^{\circ}<b<5^{\circ}$ and covers $1800 \mathrm{deg}^{2}$. This international survey is conducted not only in $\mathrm{H} \alpha$ but also makes use of two continuum filters, respectively the Sloan $r^{\prime}$ and $i^{\prime}$. IPHAS is viewed as an enhancement to former narrow-band surveys, first due to the use of CCD and the particularly small pixel scale allowed by the WFC with $0.33 \operatorname{arcsec}_{\text {pix }}{ }^{-1}$ but also (and mainly) due to the depth reached for point sources detection. Thus sources

\footnotetext{
${ }^{1}$ http: //www.iphas.org.
}

with a $r^{\prime}$ magnitude between 13 and 19.5-20 could be detected with a very good photometric accuracy. The most interesting characteristic for our purpose is the ability to detect resolved extended emissions with an $\mathrm{H} \alpha$ surface brightness down to $2 \times 10^{-17} \mathrm{erg} \mathrm{cm}^{-2} \mathrm{~s}^{-1} \operatorname{arcsec}^{-2}$.

In this paper we will focus on extended (candidate) $\mathrm{PNe}$ (i.e. objects with a size greater than $5 \operatorname{arcsec}$ ). They were searched for via a visual inspection of $2 \mathrm{deg}^{2} \mathrm{H} \alpha-r$ (continuum removal) mosaics made from the different IPHAS observations. And in order to allow the detection of objects of multiple size and brightness level, the mosaics were binned at respectively $15 \times 15$ pixels $(5 \operatorname{arcsec})$ and $5 \times 5$ pixels $(1.7$ arcsec). The first binning level, which is of particular interest to us, helps to detect resolved, low surface brightness objects (down to the IPHAS limit) and to accentuate the contours/shape of the nebulae (this is particularly useful to see, for example, the full extent of an outflow or a tail). The second set, is used to detect intermediate size nebulae i.e. smaller than $\sim 15-20$ arcsec in diameter.

The first area that has been fully investigated is the region between $R A=18 \mathrm{~h}$ and $R A=20 \mathrm{~h}$. We detected 233 candidate $\mathrm{PNe}$ among which other nebulosities may be found e.g. small Hil regions (Sabin, PhD thesis, to be published). Around $20 \%$ of this sample have been so far spectroscopically confirmed as PNe (Sabin et al., in preparation). If we look at the particularities of the $\mathrm{PNe}$ and candidate PNe uncovered, we observe that from the point of view of the size, large objects (greater than 20 arcsec) constitute the main new group (Figure 1). As large objects are generally considered as more evolved, we are confident 

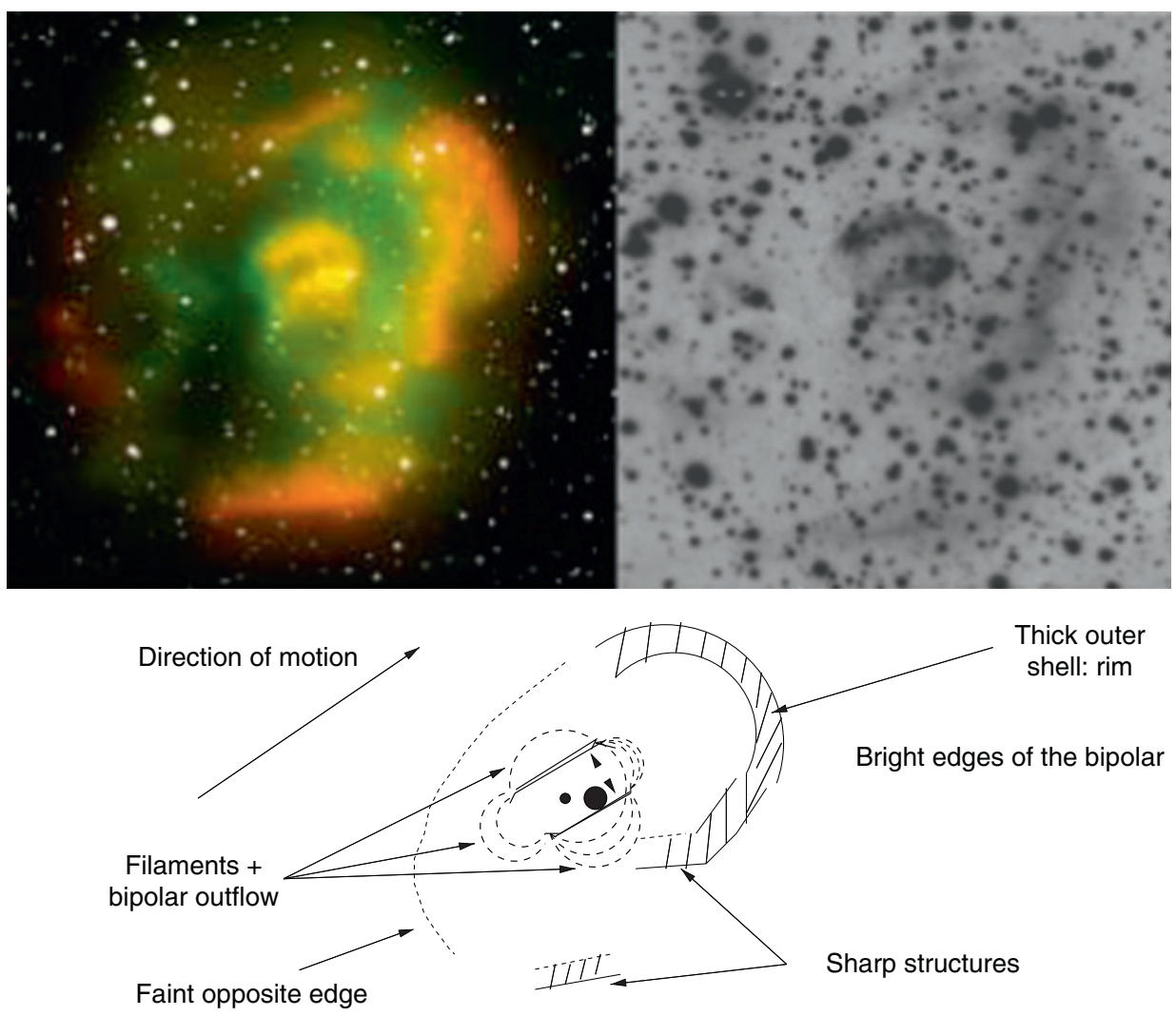

Figure 2 An example of WZO1 type: The 'Ear Nebula' IPHAS PN. North on the top and East on the left.

in finding in this group new old $\mathrm{PNe}$ and by extension new cases of PNe interacting with the surrounding ISM (PNe/ISM).

\section{Candidate PNe with ISM Interaction}

Fundamental in PN development, the interaction with the ISM does not only concern old PNe, as may be commonly thought. Indeed, the PN-ISM interaction has mainly been detected in a rather small number of nebulae, which are generally bright objects ('young' and 'midage' PNe). Rauch et al. (2000) and Wareing, Zijlstra \& O'Brien (2007) showed that different stages of interaction are exhibited during the PNe life. The low surface brightness, generally associated with nebulae mixing with the ISM and 'old' PNe, has for a long time prevented any deeper observation and good statistical study of these interactions. Although faint objects will still remain difficult to detect, the IPHAS survey provides a noticeable improvement. Nevertheless, a caveat is the difficulty to visually separate PNe-ISM from other faint and extended structures like old HII regions, Supernovae (SNRs) or diffuse $\mathrm{H} \alpha$ structures. As an example, faint bow shocks generally characteristics of PNe mixing with the ISM can also be filamentary structures from old SNRs. A spectroscopic analysis is the only way to have a clear identification.

The work presented here is based on the classification from Wareing et al. (2007) (WZO 1-4 called after the authors' names) and will allow us to establish the degree of interaction for each nebula. Their classification is the result of the first extensive investigation of the applicable parameter space, varying stellar parameters, relative velocities through the ISM and ISM densities. The depth reached by the IPHAS survey combined with the binning detection method allowed us to identify 21 cases of interacting candidate $\mathrm{PNe}$.

\subsection{WZO1 Type}

The first group of PNe/ISM concerns those where the main PN is still unaffected and which may display a distant bow shock. In our area of study $(18-20 \mathrm{~h})$, the majority of candidates answer the first condition, but none show the outer bow shock. Outside this area, the nicknamed 'Ear Nebula' or IPHASX J205013.7+465518 with a 6-arcmin size may be coincident with a WZO1 description as this object is a confirmed bipolar PN (Figure 3) surrounded by a shell which may be an AGB remnant shell or would indicate a multiple shell nebula (Figure 2).

\subsection{WZO2 Type}

This category concerns PNe showing a bright rim in the direction of motion. This is the most common feature found in our sample and 17 objects out of 21 fall under this classification. Figure 4 presents three examples with different angular sizes, although they all display a diameter on the order of a few arcmin (we considered the assumed full extent of the round nebulae). We point out in Figure 4 (top) the difficulty to determine the true 

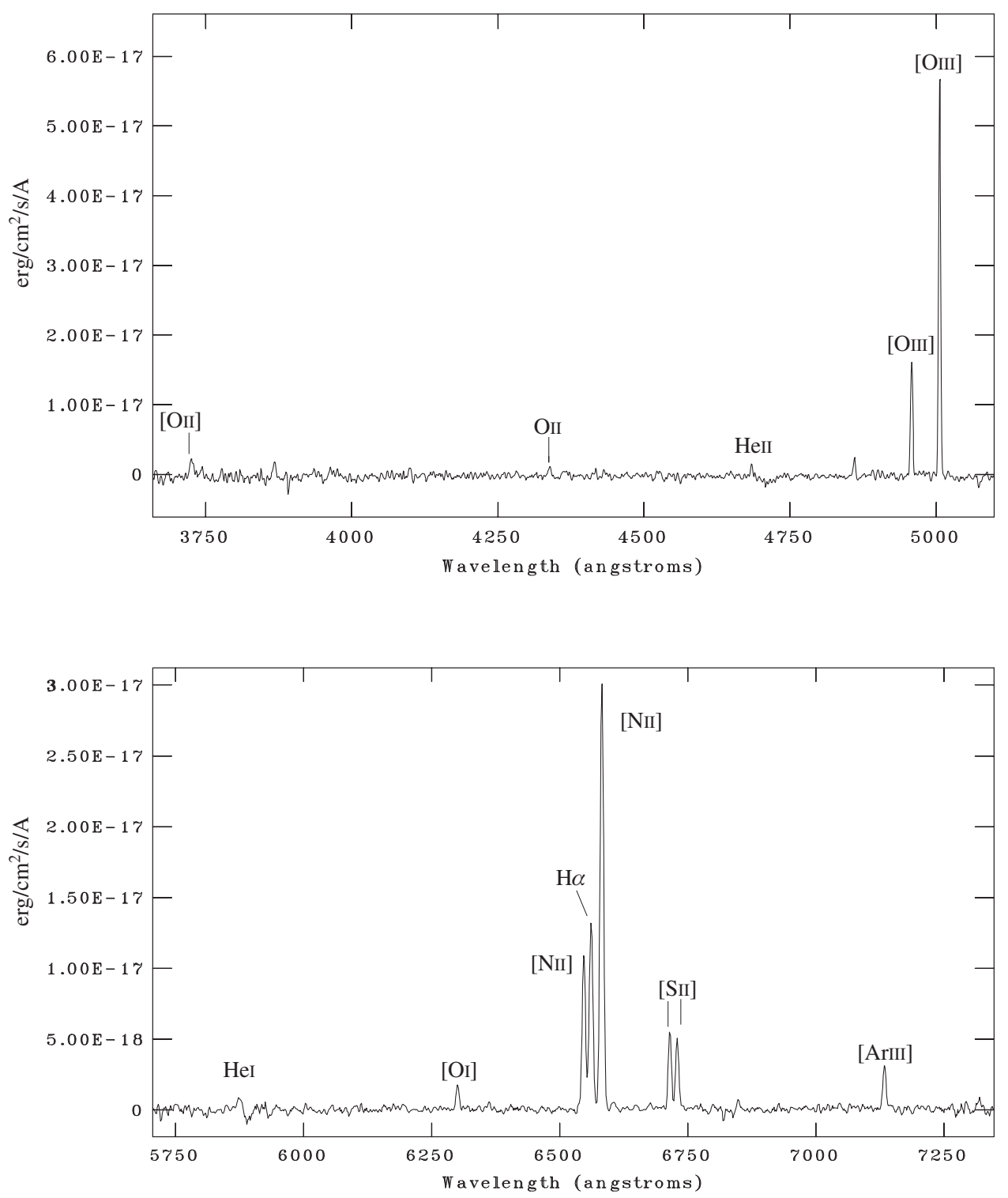

Figure 3 WHT spectra of the 'Ear Nebula' using the R300B and R158R gratings. This nebula, for which we show some of the 'strongest' emission lines useful for an identification, presents a clear [NII] over-intensity and it has been confirmed as true PN using the revised diagnostic diagram from Riesgo et al. (2006) (particularly the $\log [\mathrm{H} \alpha /[\mathrm{SII}]]$ vs. $\log [\mathrm{H} \alpha /[\mathrm{NII}]]$ diagram).

direction of motion regarding the CS position and offaxis bow shock. Such a geometry could be explained by an ISM gradient from high on the left to low on the right. We also notice a particularly low observed surface brightness $(\mathrm{SB})$ which may explain previous non detections.

\subsection{WZO3 Type}

This type is exemplified by PNe whose geometric centres are shifted away from the central star (CS): both are no longer coincident. An example, is the ancient PN Sh 2-188 around which IPHAS has uncovered an extended structure (Wareing et al. 2006). We identified 3 candidate PNe coincident with this description. The most probing WZO3 type in our sample is presented in Figure 5 and corresponds, according to hydrodynamical models, to a PN with a CS velocity of about $100 \mathrm{~km} \mathrm{~s}^{-1}$.

\subsection{WZO4 Type}

The WZO4 corresponds to the most difficult types of PN to be detected: the CS has left the vicinity of the now totally disrupted PN, leaving an amorphous structure. The challenge does not lie in the detection ability (it enters in the IPHAS range of detection) but more in the selection of the objects as possible PNe due to the total lack of symmetry or axi-symmetry. This type of interaction is also discussed in more detail by Wareing et al. in these proceedings.

We identified 1 candidate $\mathrm{PN}$ which could fit the given description. Figure 6 presents the selected candidate in the top panel. We suggest the the nebular material has been moved from the front to the rear leaving a remnant 


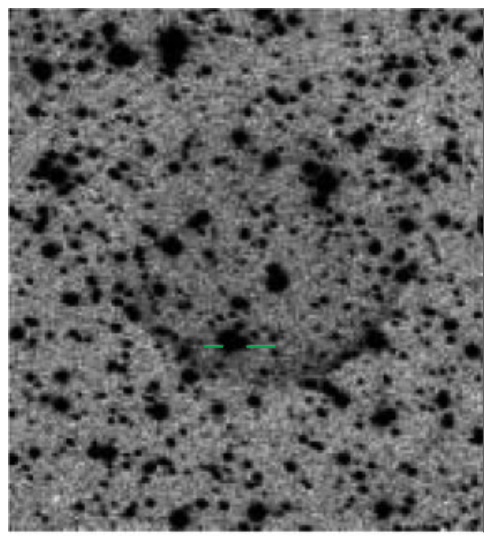

Direction of motion
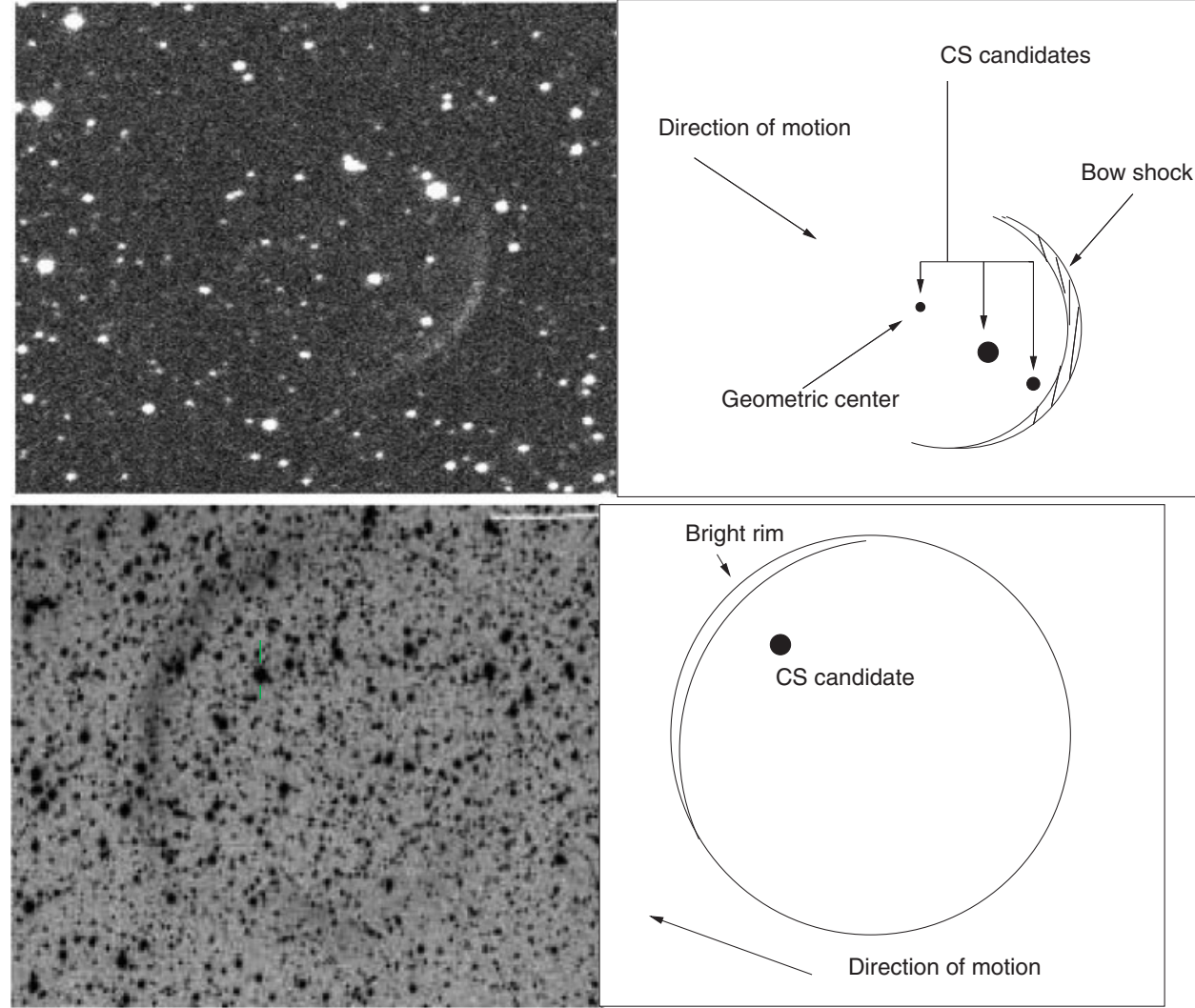

Figure 4 Examples of WZO2 types. Top: size $=1.2 \operatorname{arcmin}$ and $\mathrm{SB}=3.4 \mathrm{e}^{-17} \mathrm{erg} \mathrm{cm}^{-2} \mathrm{~s}^{-1} \mathrm{arcsec}^{-2}$. Middle: size $=8.5$ arcmin and $\mathrm{SB}=1.1 \mathrm{e}^{-16} \mathrm{erg} \mathrm{cm}^{-2} \mathrm{~s}^{-1} \operatorname{arcsec}^{-2}$. Bottom: size $=4.3 \operatorname{arcmin}$ and $\mathrm{SB}=2.7 \mathrm{e}^{-16} \mathrm{erg} \mathrm{cm}^{-2} \mathrm{~s}^{-1} \operatorname{arcsec}^{-2}$. North on the top and East on the left.

'wall of material'. We also notice that some features may be linked to turbulence effects. The comparison with the hydrodynamical model (bottom panel) seems to support this hypothesis. Nevertheless a spectroscopic confirmation of the nebula's nature will be needed. The model implies a velocity relative to the ISM of $100 \mathrm{~km} \mathrm{~s}^{-1}$ and an evolution in the post-AGB phase of 10000 years.

\subsection{Distribution of the Candidates}

Figure 7 (top) shows that the majority of the WZO2 nebulae types are located in zones of relatively low ISM density (compared to the Galactic Centre). The low stress exerted on the nebulae may explain why they still keep their quasi circular shape. The ISM is more dense in the Galactic Plane than in the zone towards the anti-centre or the zone above a height of $100 \mathrm{pc}$ (from observation of neutral hydrogen gas, Dickey et al. 1990). We therefore expected a greater influence of the interaction process in this area. Indeed, we observed that the most advanced stages of interaction, namely WZO3 and WZO4, are detected in areas of high ISM density, where PN are more likely to be affected by such densities.

The size distribution, Figure 7 (bottom), indicates that although most of the detected candidate $\mathrm{PNe}$ are $\operatorname{large} \mathrm{e}^{2}$, i.e.

\footnotetext{
${ }^{2}$ The sizes here are defined in terms of angular sizes, so the physical correspondence will depend on the distance.
} 


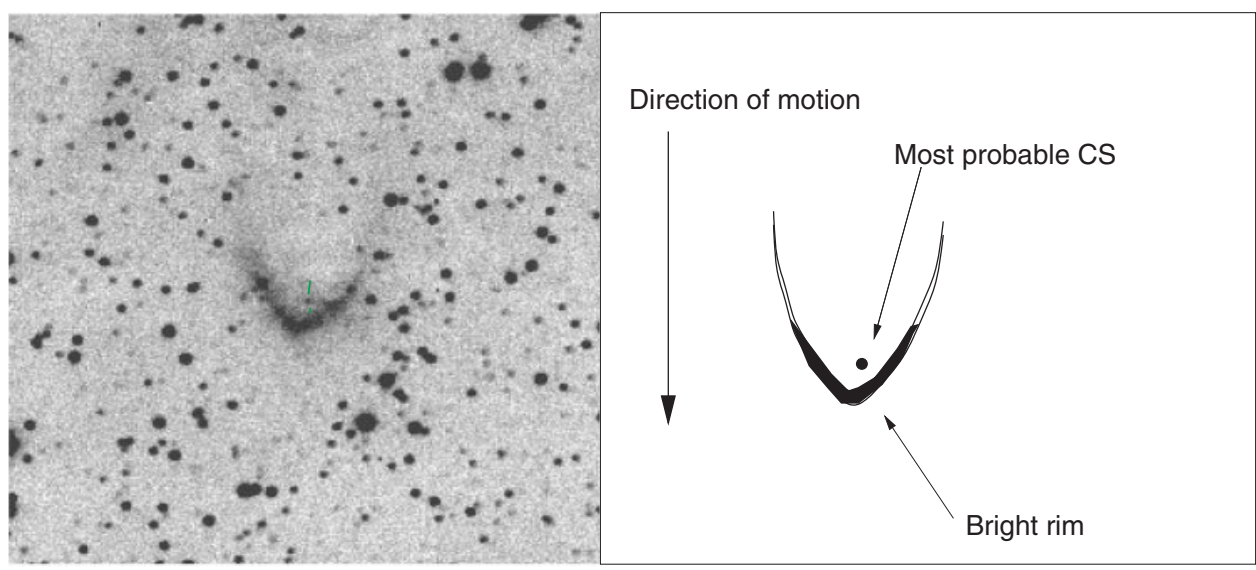

Figure 5 WZO3 type of ISM interaction in a IPHAS candidate PNe. North on the top and East on the left.

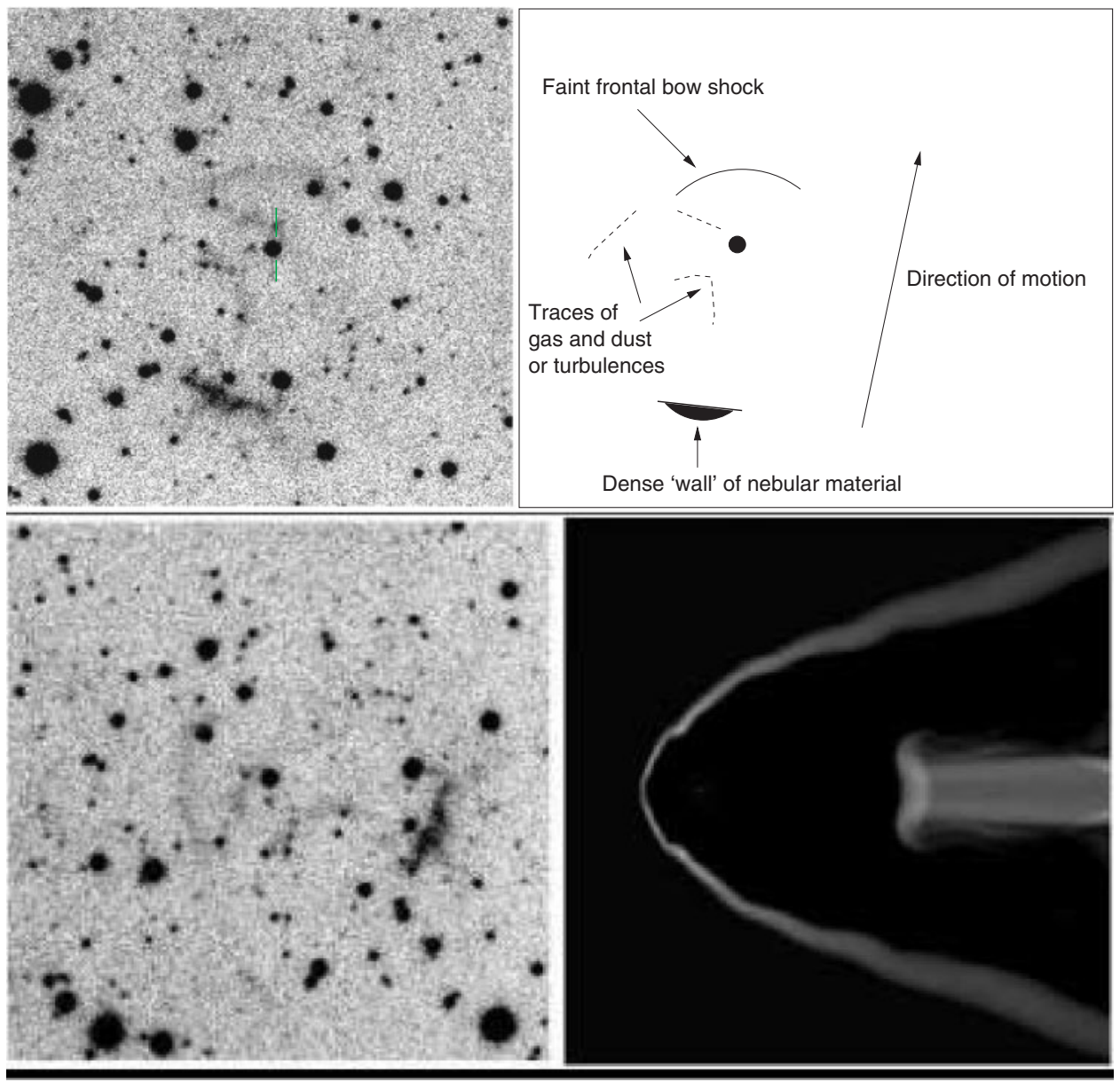

Figure 6 A possible example of WZO4 ISM interaction in one IPHAS PN candidate (top: North on the top and East on the left) with the corresponding hydrodynamical model (bottom) [reproduction of Figure 5(d) from Wareing et al. (2007)].

with a size greater than 100 arcsec, or of medium size i.e. between 20 and 100 arcsec, small nebulae also show signs of interaction. This confirms that the ISM interaction process does not 'a priori' only imply 'old' nebulae. We also observe that large objects mainly lie at higher latitudes than smaller nebulae but it is also interesting to notice that we detect large objects in zones of high extinction; large PNe seem to survive at relatively low latitudes. They would undergo strong alteration by the ISM and would display more advanced stages of interaction. Those disruptions tend to affect them more than smaller size nebulae at the same latitude range.

\section{Conclusion and Perspectives}

In the first fully analysed area of the Galactic plane, $R A=18 \mathrm{~h}$ to $R A=20 \mathrm{~h}$, the new $\mathrm{H} \alpha$ photometric survey 

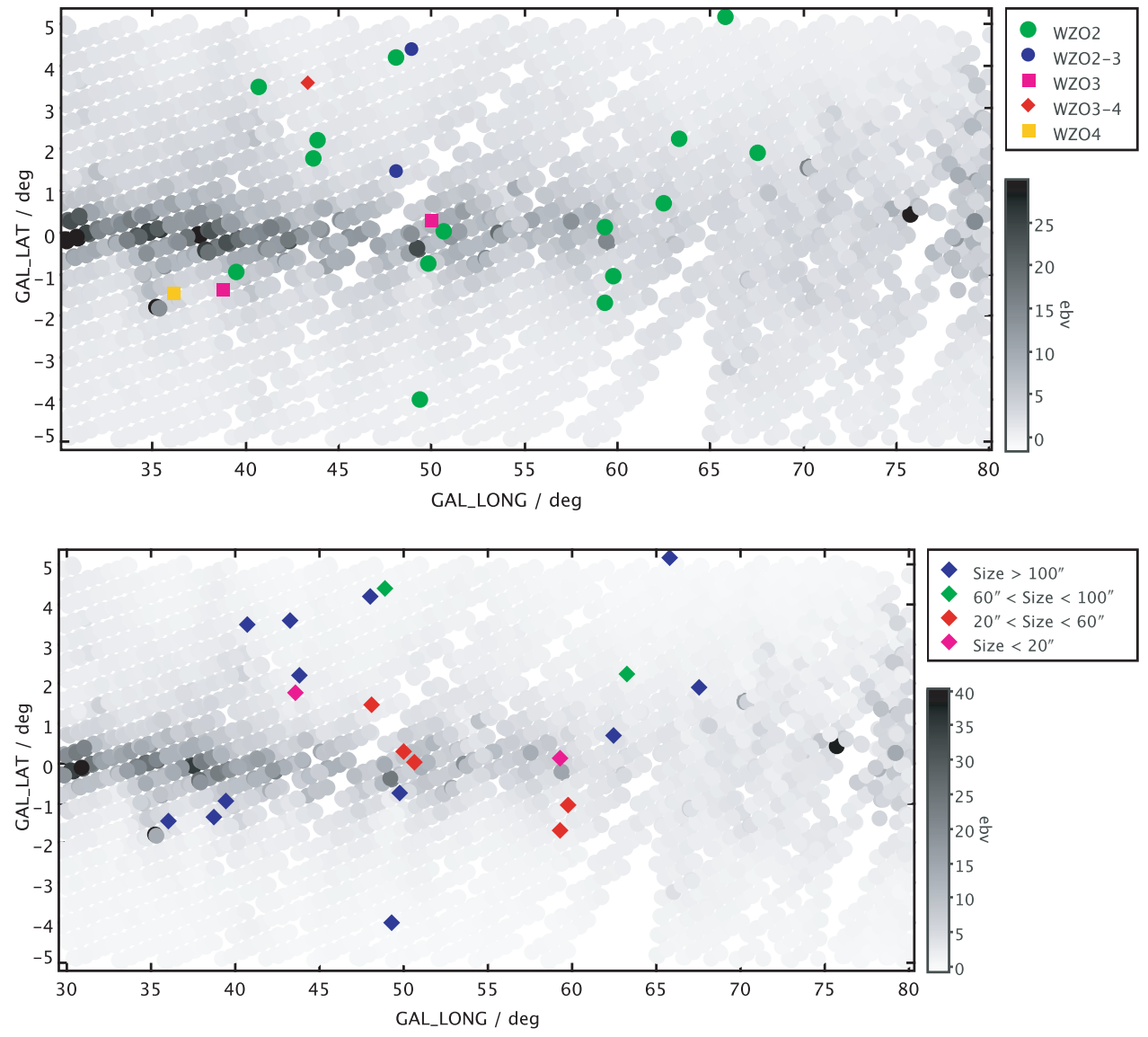

Figure 7 Galactic distribution of the candidate PNe/ISM according to their stage of interaction and their size.
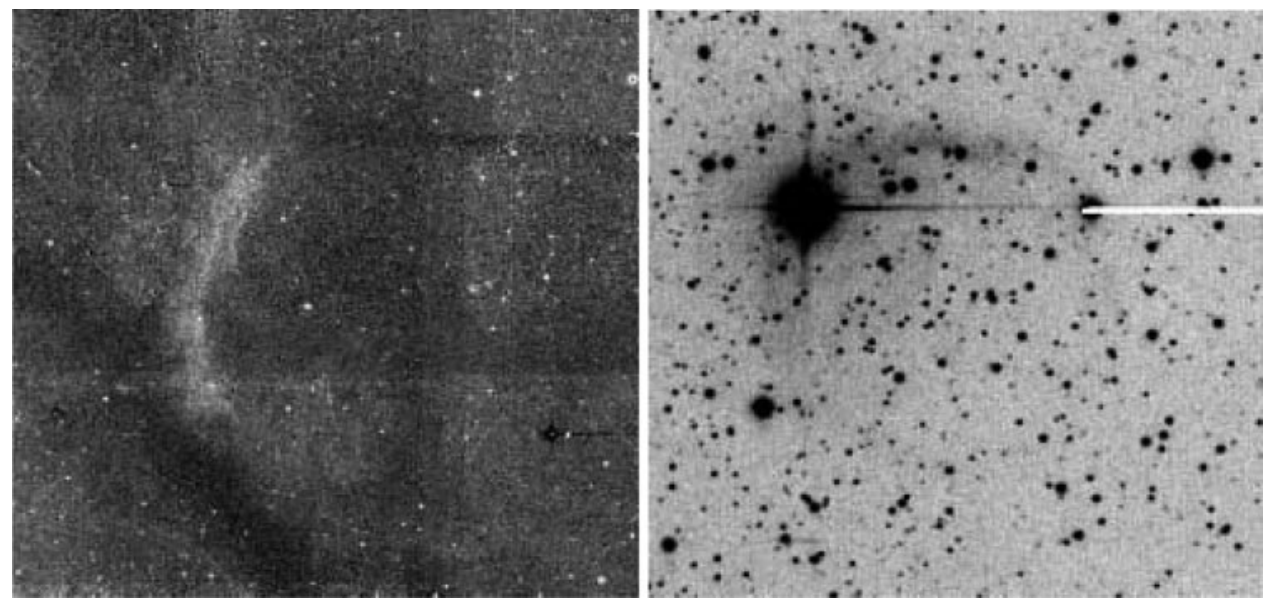

Figure 8 Example of candidates with sizes greater than $100 \operatorname{arcsec}$ (respectively 7.7 and $2.9 \operatorname{arcmin}$ ) and located at $b= \pm 1^{\circ}$. These objects present a WZO2 stage of interaction and only their (very) faint interacting rim are seen.

IPHAS appears to be an excellent tool to study PNe interacting with the ISM. Indeed the survey contributes to the detection of nebulae so far hidden mainly due to their faintness. Thus, 21 objects have been identified as possible planetary nebulae interacting with the ISM. They show diverse sizes (although the majority display a diameter greater than $100 \mathrm{arc}$ ) and morphologies corresponding to the four different cases of interaction commonly defined going from the unaffected to the totally disrupted nebula.
The most common stage is the WZO2 corresponding to nebulae showing a brightening of their rim in the direction of motion. This is coincident with the observations made by Wareing et al. (in these proceedings) crossing different $\mathrm{H} \alpha$ surveys. We were also able to reach those targets at low latitudes and found that some could survive in those environments although they would be strongly affected by the ISM. The total lack of PNe/ISM at the highest point of ISM density $\left(b= \pm 0.5^{\circ}\right.$ and $\left.30^{\circ}<l<50^{\circ}\right)$ can either be 
due to the limitation of IPHAS or because they have been totally destroyed by the effects of ISM interaction.

The next logical step is the spectroscopic identification of these sources, their central star study and physical size determination. The low surface brightness implies the use of particular means such as integral field spectroscopy to be able to retrieve the maximum information. Therefore a new programme of IPHAS PN candidate follow-up spectroscopy led by Q. Parker, A. Zijlstra and R. Corradi is now underway.

\section{References}

Acker, A., Marcout, J., Ochsenbein, F., Stenholm, B. \& Tylenda, R., 1992, Garching: European Southern Observatory, Strasbourg ESO catalogue of galactic planetary nebulae, Parts $1 \& 2$

Ali, A., El-Nawawy, M. S. \& Pfleiderer, J., 2000, APSS, 271, 245

Borkowski, K. J., Sarazin, C. L. \& Soker, N., 1990, ApJ, 360, 173 Dgani, R. \& Soker, N., 1998, ApJ, 495, 337
Dickey, J. M. \& Lockman, F. J., 1990, ARA\&A, 28, 215

Drew, J. E. et al., 2005, MNRAS, 362, 753

Gonzalez-Solares, E. A. et al., 2008, MNRAS, 388, 89

Miszalski, B., Parker, Q. A., Acker, A., Birkby, J. L., Frew, D. J. \& Kovacevic, A., 2008, MNRAS, 384, 525

Parker, Q. A. et al., 2005, MNRAS, 362, 689

Parker, Q. A. et al., 2006, MNRAS, 373, 79

Pierce, M. J., Frew, D. J., Parker, Q. A. \& Köppen, J., 2004, PASP, 21,334

Rauch, T., Furlan, E., Kerber, F. \& Roth, M., 2000, ASPC, 199, 341

Riesgo, H. \& López, J. A., 2006, RMxAA, 42, 47

Tweedy, R. W. \& Kwitter, K. B., 1996, ApJS, 107, 255

Wareing, C. J., O'Brien, T. J., Zijlstra, A. A., Kwitter, K. B., Irwin, J., Wright, N., Greimel, R. \& Drew, J. E., 2006, MNRAS, 366, 387

Wareing, C. J., Zijlstra, A. A. \& O’Brien, T. J., 2007, MNRAS, 382, 1233

Xilouris, K. M., Papamastorakis, J., Paleologou, E. \& Terzian, Y., 1996, AAP, 310, 603 